\title{
Sex Differences in Film induced Emotion: A narrative review
}

\author{
Sarika A ${ }^{1}$, Dr. Sonia George ${ }^{2}$ \\ ${ }^{1}$ Research scholar, Department of Psychology, Government College for Women University of Kerala.TVPM. \\ ${ }^{2}$ Assistant professor, Department of Psychology, Government College for Women University of Kerala.TVPM.
}

\begin{abstract}
Humankind has been fascinated by perceived differences between men and women .There are several empirical literature on sexual differences in emotion .Film is considered as one of the most effective method to induce emotions in a laboratory settings. In this study, the sex -emotion debate from the results of 24 studies which induced emotions using films in experimental settings were analysed. The differences in expressive, experiential, and physiological emotional responses of men and women collected from the studies were assessed and the key findings across three dimensions: (a) overt actions; (b) subjective reports, and (c) physiological activity were summarized.
\end{abstract}

Keywords

Emotion, Film, Mood induction, Sex, Overt actions, Subjective reports, Physiological activity

Article Received: 10 August 2020, Revised: 25 October 2020, Accepted: 18 November 2020

\section{Introduction}

Sex differences regarding emotional processing have been of great interest for years. Stereotypes about the way men and women experience and express their emotions pervade our culture and society.

Despite substantial efforts in gender differences in emotional responses over the past several decades, no consensus as to whether women are more emotional than men has been reached. Empirical studies of sex differences in emotion have produced less consistent results than the expectations based on popular convictions. Gender differences in emotional functioning are widely documented, but are often inconsistent across personality, social, cultural, and situational variables, as well as types of emotional processes, quality of emotions, and task characteristics.

Several studies have shown that females and males process emotions differently. Women have been foundto be more emotionally expressive than men possibly as a result of differences in socialization (Grossman and Wood, 1993), or the strongerpsychophysiological responses to emotional stimuli (Kring and Gordon, 1998; Orozco and Ehlers, 1998). But do gender differences actually exist between men and women, and the research is mixed regarding the emotional differences between the sexes.A fairly substantial body of research has demonstrated that women are more emotionally expressive than men; however, it remains somewhat unclear whether women also experience more emotion than men or whether they show greater physiological changes associated with emotion. Combining all ofthis information rather than relying only on self-reporting might further elucidate the findings of emotion.

The induction of an emotional state in the lab represents a long-standing issue in the investigationsof human emotions (Ray, R. D., \& Gross, J. J., 2007). There are different methods available for emotion induction, each with advantages and limitations. One of the most effective methods to study emotion is to induce emotions using films. They integrate both visual and auditory information, allowing the viewer simultaneously receives emotional cues from more than one sensory modality. Although some researchers (e.g. Albersnagel, 1988)have shown genderrelated differences for other MIPS as well, most studies have not providedfurther support for the hypothesis of gender differences.

In the present research, we aimed to increase the current knowledge of gender difference in emotion by extending the research to emotions induced byfilms.

\section{Review Method}

\section{Data search and strategy}

To form an overview of studies, we reviewed studies since 1990 until December 2019 .The primary databases include PsycINFO, Scopus research gate and EMBASE, ACM DL Medline, Web-Of-Science, PsycINFO, Cinahl, Pub Med publisher, Cochrane, and Google scholar, Sage journals online. The following search terms were used: film, movie, gender difference, sex difference, mood affect, emotion, manipulation, elicitation, evoke, measure, induction. Full papers of relevant citations were screened for relevance by two investigators (SA andSG) independently

Inclusion criteria

1) Studies which compare sex differences in induced emotion using films in an experimental design in a nonclinical sample.

2) Studies which employs a manipulation check of induced emotion using subjective report, physiological measure and overt activity.

A total of 24 studies were annotated from the journals.

\section{Results}

Table A.1 provides an overview of the studies considered for the present review. The results regarding sex differences were analysed by classifying the results based on subjective report, physiological activity, and overt expression. 
Table A.1 Sex Differences In Self Report, Physiological Response And Behavioural Expression In Film Induced Emotion

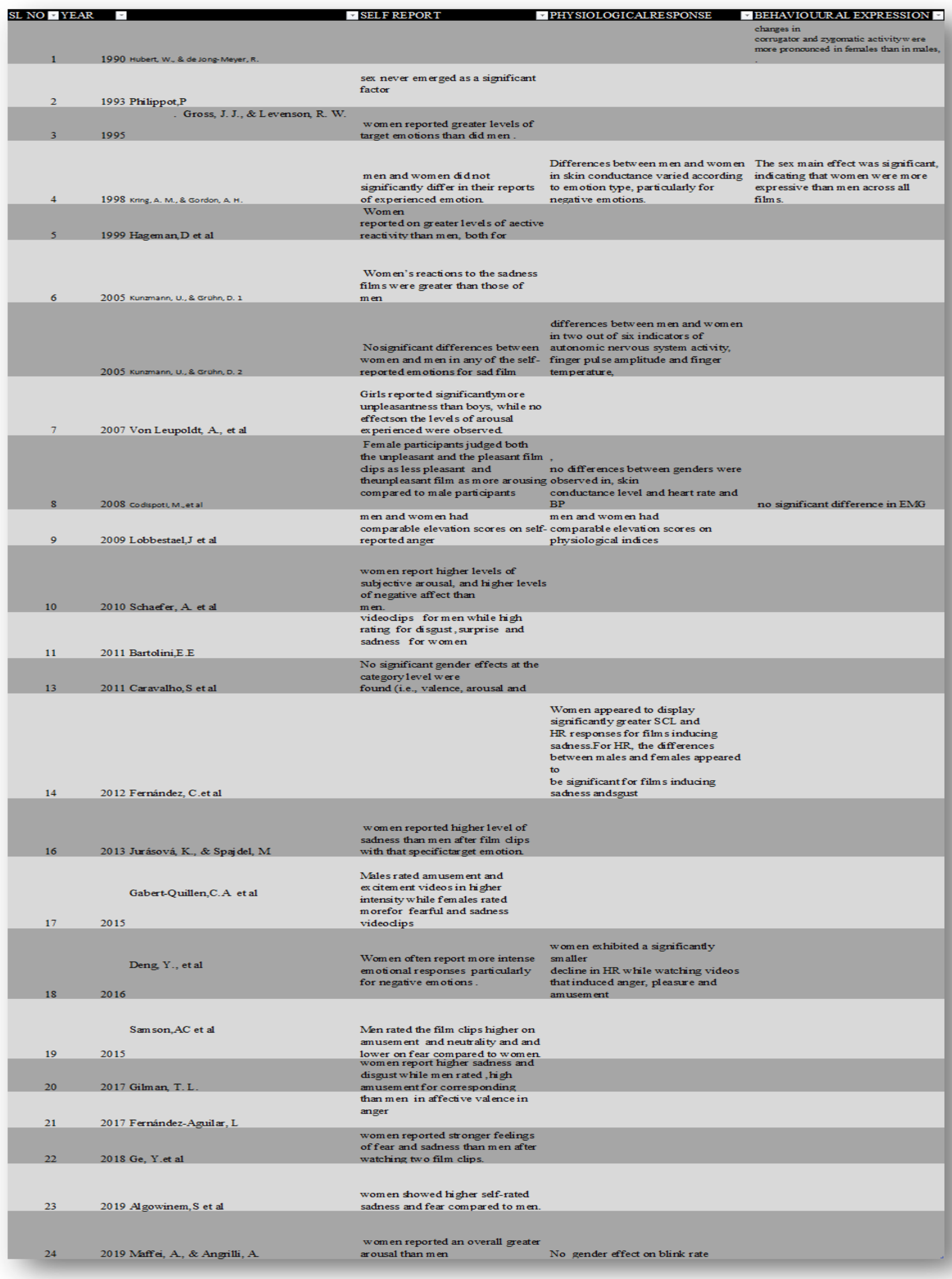




\section{Subjective report}

Gross, J. J., \& Levenson, R. W ,1995 shows that women reported greater levels of target emotions than did men .Our finding ofmore intense reports by women than men is consistent with other findings that women often report more intense emotional experiences than men(LaFrance\& Banaji, 1992; Shields, 1991). Hagemann, D., et al explored sex differences of emotional reactivity and observed greater levels of self-reported emotional reactions in women than in men, which was independent of the emotion'squality.

Von Leupoldt, A., et al (2007) observed a gender influence onaffective responses, with girls showing stronger valenceratings than boys, as reported with respect todiscrete emotions in studies by Gross and Levenson (1995). Particularly duringthe unpleasant film, girls in the present study reported significantlymore unpleasantness than boys, while no effects in the levels of arousal experienced were observed.Codispoti, (2008) foundthat Film content by Gender interaction was significant for bothpleasure and arousal ratings respectively. Female participants judged both theunpleasant and the pleasant film clips as less pleasant and theunpleasant film as more arousing compared to male participantsand physiological indices in all induction methods. Schaefer, A et al (2010) found a main effect of Gender in the arousal scale, suggesting that women reportedhigher levels of emotional arousal than men when viewing video clips. Women reported a higherNA level than men when viewing video clips. More specifically, women seemed to report higher levels of subjective arousal, and higher levels of negative affect than men. No significant gender differences werefound in positive affect as defined by the PANASscale. Studyof Fernandez-Aguilar, L (2017) revealed that women scored significantly lower than men invalence on the anger-inducing stimuli. In this sense, it was found that women present higher levels of arousal in response to clips evoking anger. Analysis of Arousal ratings in the studies of Maffei, A., \& Angrilli, A. (2019) showed a significant main effect of Sex and of Film. Women reported an overall greater arousal than men over all for emotional clips. Arousal evaluations by Maffei, A., \& Angrilli, A., (2019) showed a main effect in which women reported an overall greater arousal than men; this difference was particularly evident for unpleasant clips, especially fear. The results of Deng, Y., et al, (2016) also showed that women reported a higher arousal value than did men on most emotiontypes. Women often report more intense emotional responses particularly for negative emotions. Women also reported lower valence, higher arousal, and stronger avoidance motivation.

Bartolini, E. E. (2011) showed that emotion ratings for excitement differed significantly by participant gender, with men tending to rate slightly higher levels of excitement across all film clips .Results for disgust and surprise also approachedsignificance with women rating both emotions slightly higher thanmen across all film clips. Women reacted with higher level of sadness to all film clips with that specific target emotion on the studies of Jurásová, K., \&Spajdel (2013). Gabert-Quillen, C. A.,(2015)found that significant gender differences exist for some of the video clips for targeted emotion in which males rated excitement and amusement video clips with higher intensity for the target emotion while females rated fearful and sad clips as more fearful and sadder than males. Samson, AC et al, (2015) found significant difference in rating of some for some emotion to women compared to men. Men rated the film clips higher on amusement and neutrality and lower on fear compared to women. Significant sex difference in rating for sadness, disgust and amusement can also be seen in the studies of Gilman, T. L.,(2017) in which women reported higher sadness and disgust for sadness and disgust eliciting stimuli while men rated high on high amusement stimuli. Studies of Algowinem, S et al, (2019) shows that Women showed higher self-rated sadness and fear compared to men, which was expected basedon gender-differences in emotion studies. The studies showed that women were more expressive for weak emotions (e.g., fear, sadness), while men showed strong emotion (e.g., anger). Moreover, women self-rating for the Arabic disgust clip differed between the genders, where women ratedhigher disgust, while men rated higher amusement. Similar results of high rating of fear and sadness in women can be seen in studies of Ge, Y., (2019) where women reportedstronger feelings of fear and sadness than men afterwatching the film clips. Such gender differenceswere also reported in other studies that used film clips intending to elicit different emotional states. (Gross\& Levenson, 1995; Hagemann et al., 1999; Schaefer et al., 2010).

Early Studies of film induced emotion of Philippot, P, 1993 shows that sex never emerged as a significant factor, male and female participants responded in the same way to the films Men and women did not significantly differ in their reports of experienced emotion. No significant gender effects at the valence, arousal and dominance were found in the studies of Caravalho, $\mathrm{S}$ et al (2011). There is limited evidence for gender differences in subjective reactivity in the studies of Kring, A. M., \& Gordon, A. H.(1998). Lobbestael, J (2008) demonstrated that men and women had comparable elevation scores on self-reported anger

\section{Physiology}

Studies reported gender differences for physiological responding, namely, skin conductance level and heart rate. From the studies of Kring, A. M., \& Gordon, A. H., 1998, it is found that differences between men and women in skin conductance varied according to emotion type, particularly for negative emotions. That is, men had greater reactivity to fear and anger films, and women had greater reactivity to sad and disgust films.

Fernandez, C et al (2012) found that Variations in SCL were significantly different between males and females for sad films, where women had more intense SCL scores than men .With regard to $\mathrm{HR}$, the differences between males and females appeared to be significant for films inducing 
sadness and disgust being the females more reactive than males.

Kunzmann, U., \& Grühn, D. (2005) found differences between men and women in two out of six indicators of autonomic nervous system activity, finger pulse amplitude and finger temperature, suggesting greater physiological reactivity in women than in men.

Regarding the gender differences in HR, Deng, Y., et al, 2016 found that women exhibited a significantly smaller decline in HR while watching videos that induced anger, pleasure and amusement.

While other three studies show no difference in physiological activity. Codispoti, (2008) found that no differences between genders were observed in skin conductance level and heart rate. Studies of Lobbestael, J., (2008) demonstrated that men and women had comparable elevation scores on BP, Heart rate and SCL for anger.Maffei, A., \& Angrilli, A., (2019) shows that there is no sex difference in blink rate even though there is clear difference in subjective rating

\section{Expression of emotion}

The present study of Kring, A. M., \& Gordon, A. H., 1998 found that women were more expressive than men.That is, women exhibited more positive expressions in responseto happy films and more negative expressions in response tosad and fear films.Studies using facial electromyography activity show inconsistent results regarding the sex differencesin expression of emotion.In the studies of Hubert, W., \& de Jong-Meyer, R., 1990 females exhibited more CS EMGthan males, changes in corrugator and zygomatic activity were more pronounced in females than in males. A contradictory result from Codispoti, 2008 shows that no differences between genders were observed in EMG activity.

\section{Discussions}

The present study explored gender differences in emotional experience, physiological responses and emotional expressivity in film induced emotion.

Self-report measures, are the basis of much of the available evidence about emotion and we explored sex differences of emotional reactivity and observed greater levels of selfreported emotional reactions in women than in men, which was independent of the emotion's quality. The result from the studies of Gross and Levenson (1995) who reported on more intense emotion reports by women than men is consistent with other findings of sex differences in the experience of emotions (Shields, 1991; Brody\& Hall, 1993).A higher level of reported emotionalfeelings for female participants is consistent in studies of Gross and Levenson's (1995) and Hagemanet al.'s (1999), Deng, Y., et al (2016).These results may reflect either neurobiological differences in emotional systems across genders (Cahill, Uncapher, Kilpatrick, Alkire, \& Turner, 2004) or the effects of socio-cultural stereotypesabout gender roles (Brody \& Hall, 2000; Feldman-Barrett, Robin, Pietromonaco, \& Eyssel, 1998; Kelly \& Hutson-Corneaux, 1999)

Someof the studies of gender differences using films were based on the use of emotional stimuli within the broader classification of dimensions such as pleasant and unpleasant categoriesor, higharousal and low arousal .Women reported an overall greater arousal than men foremotional film clips. This difference was particularly evident for unpleasant clips. This is in line with current literature on both emotional slides and clips showing a greater sensitivity of women compared to men to emotional stimulation in general and especially to unpleasant contents (Bianchin \& Angrilli, 2012;Bradley, Codispoti, Sabatinelli, \& Lang, 2001; Maffei et al., 2011).Findings suggest that across film clips, females react more strongly to negative emotions than do males, Additionally, males react more strongly to positive emotions than do females, and they also tend to attribute positive emotions to negatively valence films.A similar gender effect on valence ratings but not on arousal ratings during unpleasant emotional stimulation has been reported in a study by Sharp et al. (2006) in children of comparable age who were stimulated with affective pictures. Results are consistent with the interpretation that females rather than males are more susceptible to negative life experiences and lowered mood, and may have implications for the pathophysiology of mood disorders such as depression.

Some studies focus the responses of men and women for discrete emotions also. The specific emotions reported more intensely or more frequently by women include sadness, fear, and disgust.This greater response of fear and sad response in women is consistent with other studies (Scherer, Walbotlin, \& Summerfield, 1986). Another notable feature is the higher intensity response of man towards excitement and amusement emotions. Males rated amusement and excitement videos in higher intensity while females rated more for fearful and sadness and disgust videoclip.The results from analysing the sex differences on discrete emotions are consistent to that of the results from dimensions of emotion such thatfemales react more strongly to negative emotions than do males and males react more strongly to positive emotions than do females. No consistent results in higher rate of anger in men can be observed in response to anger eliciting video clips. Reports of anger, however, have not yielded consistent sex differences. Although men may express more anger through vocal, facial, and behavioural modalitiesthan women, the data on gender differences in anger from research using selfreportquestionnaires are inconsistent.

Researchers who wish to elicit emotions in their participants should be aware that these differences are not limited to specific emotions, and that the content of certain scenes in film clips may also produce different effects for males than for females. This may have been due to the gender-relevant content in the film.

There are also opposite findings across the studies about gender differences in emotional reactivity to films. The possible reason could be the fact, that each study used different film excerpts. It would be useful to assess what kind of plot, visual and verbal factors can influence emotional reactions in men andwomen.

\section{Physiological responses}

Studies of the sex differences in physiologicalresponses of emotion reveal a complex and incomplete picture because of the limited number of physiological studies and of the 
inconsistent results available from these studies. There is a pattern of mixed results when gender is considered.

Significant result between men and women shows that there is significant difference in terms of certain emotions. However, present studies reported gender differences for other indicators of physiological responding namelyskin conductance level (Kring \& Gordon, 1998) andheart rate (Kunzmann, U., \& Grühn, D., 2005. In sum, this evidence support neither general nor robust gender differences physiological reactivity. In spite of gender differences in subjective reports, men and women are quite similar in their affective physiological reactions to pleasant and unpleasant stimuli (Bradleyet al., 2001b; Caseras et al., 2007; Frazier et al., 2004; Kreibig et al.,2007; Sanchez-Navarro and Martinez-Selva, 2006; Wrase et al., 2003. Future studies should adopt more indicators, of physiological response. While three studies shows comparable elevation scores on physiological indices in men and women (Lobbestael, J., 2008; Maffei, A., \& Angrilli, A., 2019; Codispoti, 2008). Other studies also show greater reactivity of men to certain types of emotion (Deng, Y., et al, 2016; Kring, A. M., \& Gordon, A. H., 1998). This may mean that men are aroused internally, but they restrict their emotions whereas women freely express emotions, as proposed by Buck and others (Buck, 1977, 1984; Levensen, Carstensen, \& Gottman, 1994)

\section{Expression of emotion}

Several research studies and meta-analyses have shown that there is significant gender differences in the expression of emotion, with women showing greater emotion expression overall (Brody\& Hall, 1993). It is important to note that these behaviours do not have to reflect emotional states (Krauss, Chen, \& Chawla, 1996). A possible explanation is that both men and women may be reinforced by social and cultural standards to express emotions differently, but it is not necessarily true in terms of experiencing emotions. For instance, studies suggest that women often occupy roles that conform to feminine display rules, which require them to amplify their emotional response to impress others .(Derks, Daantje; Fischer, Agneta H.; Bos, Arjan E.R. 2008). Except the study of Codispoti, (2008) in which no differences between genders were observed in EMG activity, other two studies shows that there is significant difference between male and female terms of emotional expression. Study by Hubert, W., \& de Jong-Meyer, R, 1990 found that changes in corrugator and zygomatic activity were more pronounced in females than in males. Consistent with a number of previous investigations, study of Kring, A. M., \& Gordon, A. H., 1998 found that women were more expressive than men. Gender role has been posited as a possible contributor to sex differences in expressivity ( Brody \& Hall, 1993)

In addition to assessing men's and women's overall levels of emotional response on self-report and physiological measures, we also evaluated the correspondence between these three indicators. Ifsex differences in emotional intensity are innate, then correspondence should be found across the multiple assessments of emotional response for both sexes. That is, women should generate more intense emotions than men on both self-report measures, physiological response and EMG assessments of muscle response. Furthermore, on a correlational basis, members of both sexes reporting intense emotions should display intense physiological reactions, whereas those reporting mild emotional reactions should display moderate physiological responses. The degree to which the expressive, experiential, and physiological components of emotion correspond to one another varies depending on a number of social, cultural, and situational factors (Ekman, Friesen, \& Ellsworth, 1982; Lang, Bradley, \& Cuthbert, 1990; Miller \& Kozak, 1993).Most of the time gender differences were only expressed in the self-report, no such physiological experiences are not evident and there is inconsistent pattern of overt expression also. Compared to men, women rated emotional films as more arousing in reporting emotion. These findings are not supported by the physiological measures: even if women rated both emotional films as less pleasant and the unpleasant film as more arousing than men, no differences between genders were observed in skin conductance level and heart rate (Maffei, A., \& Angrilli, A.,2019; Deng, Y., et al,2016; Codispoti, M.,et al,2008.). Men and women are quite similar in their affective physiological reactions to pleasant and unpleasant stimuli (Bradley et al., 2001; Kreibig et al., 2007). The comparison of physiological arousal, experience and expression of emotion will give much insight about the stereotypes andthe emotion regulation strategy of individuals. The participants in these studies may have regulated their emotions while watching the emotional videos. Although they were asked to feel their emotions, the possibility of emotion regulation cannot be excluded. The gender differences in emotional responses may be due to the gender differences in emotional regulation. Kring andGordon (1998) documented gender differences in facial expressions, but not in self-reports of experience, in response to evocative films. Even when the women did not experience particularly strong negative emotion, they might have regulated their emotions, interpreting them as more negative, which might explain why their self-report was more intense for emotions such as sadness, horror and disgust. It is acknowledged that examining self-report, physiological activity and expression allows for an understanding of gender differences in both the cognitive and emotional domains. Research has often relied exclusively on subjective reports to measure andassess emotions. When observed, such sex differences tend to be either context dependent (Vogel, Tucker, et al., 1999) or influenced by learned gender roles (Gilligan, 1993) rather than reflective of basic, innate differences in affective ability. Future research should use more observational and physiological measures to enhance the results of self-report research.

Taken together, it appears that sex irrespective of the stimulation technique employed might be an important source of individual differences in emotions. However, the gender differences that appear on self-report measures with females reporting intense emotions than men are unlikely to be solely determined by stereotypes, self-presentation biases, memory encoding, or other cognitive differences between males and females are also holds responsibility.

\section{Conclusion}


The present study contributes to an expanded literature concerning the sex differences in emotion. Women tend to report stronger emotional experiences than men for subjective report and inconsistent and limited studies are available for interpreting the sex differences in physiological and expression of emotion in response to films. Poor correspondence between subjective report physiological response and expression in sex difference fails to unequivocally support the idea that men and women are innately emotionally different.

\section{References}

[1] Albersnagel, F. A. (1988). Velten and musical mood induction procedures: A comparison with accessibility of thought associations. Behaviour research and therapy, 26(1), 79-95.

[2] Alghowinem, S., Goecke, R., Wagner, M., \& Alwabil, A. (2019). Evaluating and validating emotion elicitation using English and Arabic movie clips on a Saudi sample. Sensors, 19(10), 2218

[3] Bartolini, E. E. (2011). Eliciting emotion with film: Development of a stimulus set.

[4] Bradley, M. M., Codispoti, M., Sabatinelli, D., \& Lang, P. J. (2001). Emotion and motivation II: sex differences in picture processing. Emotion, 1(3), 300.

[5] Brody, L. R., \& Hall, J. A. (1993). Gender and emotion. handbook of emotions.

[6] Carvalho, S., Leite, J., Galdo-Álvarez, S., \& Gonçalves, O. F. (2012). The emotional movie database (EMDB): A self-report and psychophysiological study. Applied psychophysiology and biofeedback, 37(4), 279-294.

[7] Codispoti, M., Surcinelli, P., \& Baldaro, B. (2008). Watching emotional movies: Affective reactions and gender differences. International Journal of Psychophysiology, 69(2), 90-95.

[8] Deng, Y., Chang, L., Yang, M., Huo, M., \& Zhou, R. (2016). Gender differences in emotional response: Inconsistency between experience and expressivity. PloS one, 11(6), e0158666

[9] Derks, Daantje; Fischer, Agneta H.; Bos, Arjan E.R. (2008). "The role of emotion in computer-mediated communication: A review". Computers in Human Behaviour. 24 (3): 766-785. doi:10.1016/j.chb.2007.04.004.

[10] Ekman, P., Friesen, W. V., \& Ellsworth, P. (1982). What are the similarities and differences in facial behavior across cultures. Emotion in the human face, 2, 128-44.

[11] Fernández-Aguilar, L., Ricarte, J., Ros, L., \& Latorre, J. M. (2018). Emotional differences in young and older adults: Films as mood induction procedure. Frontiers in psychology, 9, 1110

[12] Fernández, C., Pascual, J. C., Soler, J., Elices, M., Portella, M. J., \& FernándezAbascal, E. (2012). Physiological responses induced by emotion-eliciting films. Applied psychophysiology and biofeedback, 37(2), 73-79.

[13] Gabert-Quillen, C. A., Bartolini, E. E., Abravanel, B. T., \& Sanislow, C. A. (2015). Ratings for emotion film clips. Behaviour research methods, 47(3), 773787.

[14] Ge, Y., Zhao, G., Zhang, Y., Houston, R. J., \& Song, J. (2019). A standardised database of Chinese emotional film clips. Cognition and Emotion, 33(5), 976-990.

[15] Gilligan, C. (1993). In a different voice: Psychological theory and women's development. Harvard University Press.

[16] Gross, J. J., \& Levenson, R. W. (1995). Emotion elicitation using films. Cognition \& emotion, 9(1), 87-108.

[17] Grossman, M., \& Wood, W. (1993). Sex differences in intensity of emotional experience: a social role interpretation. Journal of personality and social psychology, 65(5), 1010.

[18] Hagemann, D., Naumann, E., Maier, S., Becker, G., Lürken, A., \& Bartussek, D. (1999). The assessment of affective reactivity using films: Validity, reliability and sex differences. Personality and Individual differences, 26(4), 627-639.

[19] Hall, J. A., Carter, J. D., \& Horgan, T. G. (2000). Gender differences in nonverbal 
communication of emotion. Gender and emotion: Social psychological perspectives, 97-117.

[20] Hubert, W., \& de Jong-Meyer, R. (1990). Psychophysiological response patterns to positive and negative film stimuli. Biological psychology, 31(1), 73-93.

[21] Jurásová, K., \& Spajdel, M. (2013). Development and assessment of film excerpts used for emotion elicitation. Activitas Nervosa Superior Rediviva, 55(3), 135-140.

[22] Krauss, R. M., Chen, Y., \& Chawla, P. (1996). Nonverbal behavior and nonverbal communication: What do conversational hand gestures tell us? In Advances in experimental social psychology (Vol. 28, pp. 389-450). Academic Press

[23] Kreibig, S. D., Wilhelm, F. H., Roth, W. T., \& Gross, J. J. (2007). Cardiovascular, electrodermal, and respiratory response patterns to fear-and sadness-inducing films. Psychophysiology, 44(5), 787-806

[24] Kring, A. M., \& Gordon, A. H. (1998). Sex differences in emotion: expression, experience, and physiology. Journal of personality and social psychology, 74(3), 686.

[25] Kunzmann, U., \& Grühn, D. (2005). Age differences in emotional reactivity: the sample case of sadness. Psychology and aging, 20(1), 47.

[26] LaFrance, M., \& Banaji, M. (1992). Toward a reconsideration of the genderemotion relationship. Emotion and social behavior, 14, 178-201.

[27] LaFrance, M., \& Hecht, M. A. (2000). Gender and smiling: A meta-analysis. Gender and emotion: Social psychological perspectives, 118-142.

[28] Lane RD, Quinlan DM, Schwartz GE, Walker PA and Zeitlin SB. The Levels of Emotional Awareness Scale: a cognitivedevelopmentalmeasure of emotion. J Pers Assess 1990; 55:124-134

[29] Lang, P. J., Bradley, M. M., \& Cuthbert, B. N. (1990). Emotion, attention, and the startle reflex. Psychological review, 97(3), 377.

[30] Lobbestael, J., Arntz, A., \& Wiers, R. W. (2008). How to push someone's buttons: A comparison of four anger-induction methods. Cognition \& Emotion, 22(2), 353-373.

[31] Maffei, A., \& Angrilli, A. (2019). Spontaneous blink rate as an index of attention and emotion during film clips viewing. Physiology \&behaviour, 204, 256-263.

[32] Miller, G. A., \& Kozak, M. J. (1993). Three-systems assessment and the construct of emotion. The structure of emotion, 31-47

[33] Orozco, S., \& Ehlers, C. L. (1998). Gender differences in electrophysiological responses to facial stimuli. Biological psychiatry, 44(4), 281-289.

[34] Overbeek, T. J., van Boxtel, A., \& Westerink, J. H. (2012). Respiratory sinus arrhythmia responses to induced emotional states: effects of RSA indices, emotion induction method, age, and sex. Biological psychology, 91(1), 128-141.

[35] Philippot, P. (1993). Inducing and assessing differentiated emotion-feeling states in the laboratory. Cognition and emotion, 7(2), 171-193.

[36] Quigley, K. S., Barrett, L. F., \& Weinstein, S. (2002). Cardiovascular patterns associated with threat and challenge appraisals: A within-subjects analysis. Psychophysiology, 39(3), 292-302

[37] Ray, R. D., \& Gross, J. J. (2007). Emotion elicitation using films. Handbook of emotion elicitation and assessment, 9.

[38] Samson, A. C., Kreibig, S. D., Soderstrom, B., Wade, A. A., \& Gross, J. J. (2016). Eliciting positive, negative and mixed emotional states: A film library for affective scientists. Cognition and Emotion, 30(5), 827-856.

[39] Schaefer, A., Nils, F., Sanchez, X., \& Philippot, P. (2010). Assessing the effectiveness of a large database of 
emotion-eliciting films: A new tool for emotion researchers. Cognition and emotion, 24(7), 1153-1172.

[40] Vogel, D. L., Tucker, C. M., Wester, S. R., \& Heesacker, M. (1999). The impact of sex and situational cues on the endorsement of traditional gender-role attitudes and behaviors in dating couples. Journal of Social and Personal Relationships, 16(4), 459-473.

[41] Von Leupoldt, A., Rohde, J., Beregova, A., Thordsen-Sörensen, I., Zur Nieden, J., \& Dahme, B. (2007). Films for eliciting emotional states in children. Behaviour Research Methods, 39(3), 606-609. 